\title{
Embryonic lateral inhibition as optical modes: An analytical framework for mesoscopic pattern formation
}

\author{
Jose Negrete, Jr." and Andrew C. Oates \\ Institute of Bioengineering, School of Life Sciences and School of Engineering, École Polytechnique Fédérale de Lausanne (EPFL), \\ CH-1015 Lausanne, Switzerland
}

(Received 9 October 2018; published 25 April 2019)

\begin{abstract}
Cellular checkerboard patterns are observed at many stages of embryonic development. We study an analytically tractable model for lateral inhibition and show that the steady states are analogous to optical phonons at the $\Gamma$ point, which have the wave number $k=0$. We study the cases of cells arranged in linear and hexagonal lattices. To determine how the final pattern is selected it is necessary to take into account the granularity of the pattern and, analogously to solid-state physics, to redefine the basis and lattice sites in terms of a periodic crystal. The sites and basis are determined by looking at the symmetries of inhibitory interactions between cells. The redefined basis for cells placed in a linear lattice is composed by two cells which are embedded in another linear lattice, while for cells placed in a hexagonal lattice the redefined basis consists of three cells embedded in another hexagonal lattice. The pattern in hexagonal lattices can be driven into three different states: two of those states are periodic checkerboards and a third in which both periodic states coexist. These observations provides new predictions for experiments.
\end{abstract}

DOI: 10.1103/PhysRevE.99.042417

\section{INTRODUCTION}

Pattern formation in living tissue is an emergent property that arises from cell signaling. In his seminal work Alan Turing proposed that the anatomical structure of an embryo is determined by self-organized chemical patterns [1]. In his theory the interplay between reactions and diffusion of two chemical species gives rise to self-organized periodic structures. Although this model is difficult to implement in practice with the restrictions proposed by Turing [2,3], variants of this model system have been extensively studied theoretically [4] and have been successfully used to describe the patterns of living systems at the tissue level [5-7].

There is a subclass of patterns in living systems where the characteristic length scale $(\lambda)$ is the size of two cells [Figure 1(a)]. These are fine-grained patterns where protein expression levels vary abruptly and regularly between cells, reminiscent of checkerboards [Fig. 1(a)]. These patterns appear in several examples of cellular tissues such as in the arrangement of photoreceptor cells in the eye [8], sensory hair cells in the auditory epithelium $[9,10]$, and sensory bristles in the fly thorax [11] among others. Lateral inhibition has been proposed as a basic mechanism whereby a cell inhibits the expression levels of a protein in its neighboring cells. This is in contrast to Turing patterns where the pattern is created by the negative feedback between the fast- and slow-diffusing components. The Delta-Notch signaling

\footnotetext{
*jose.negretejr@epfl.ch
}

Published by the American Physical Society under the terms of the Creative Commons Attribution 4.0 International license. Further distribution of this work must maintain attribution to the author(s) and the published article's title, journal citation, and DOI. system is a pathway frequently used by different species to create these fine-grained tissue patterns [12].

Collier et al. [13] pioneered theoretical work in lateral inhibition, analyzing a spatially discrete model for DeltaNotch signaling that reproduced periodic and fine-grained patterns. Since then, extensions of this and similar models have incorporated different effects observed in experiments, such as self-regulation (cis interactions) [14], state-dependent coupling strength [15], time delay in signaling [16,17] and cis interactions with time delay [18]. These models are difficult to analyze analytically and they rely on numerical simulations to determine how patterns emerge. In this work we propose a generic model for lateral inhibition that is analytically tractable, allowing us to understand pattern selection for this class of systems. The model consists of the following equation:

$$
\frac{d u_{n}}{d t}=\underbrace{-u_{n}-\gamma u_{n}^{3}+\Omega_{n}}_{\text {cis regulation }}+\underbrace{\epsilon \sum_{i=1}^{N} u_{n}^{(i)}}_{\text {trans regulation }},
$$

where the state of each cell placed in a lattice is represented by the variable $u_{n}$ (e.g., the amount of active delta). The model describes the effects of $c i s$ interactions where $\Omega_{n}$ (e.g., the source of active delta) is an internal component that influences the production of $u_{n}$ and $\gamma>0$ is the strength of nonlinear degradation. The cells are influenced by the state of their $N$ nearest neighbors $u_{n}^{(i)}$ (trans regulation) with coupling strength $\epsilon$ [Fig. 1(b)]. The regime where $\epsilon<0$ corresponds to lateral inhibition.

We can write the continuum approximation for Eq. (1) for cells placed in a linear lattice,

$$
\begin{aligned}
\frac{\partial u(x, t)}{\partial t}= & (2 \epsilon-1) u(x, t)-\gamma u^{3}(x, t) \\
& +\epsilon \Delta x^{2} \frac{\partial^{2} u(x, t)}{\partial x^{2}}+\Omega(x),
\end{aligned}
$$




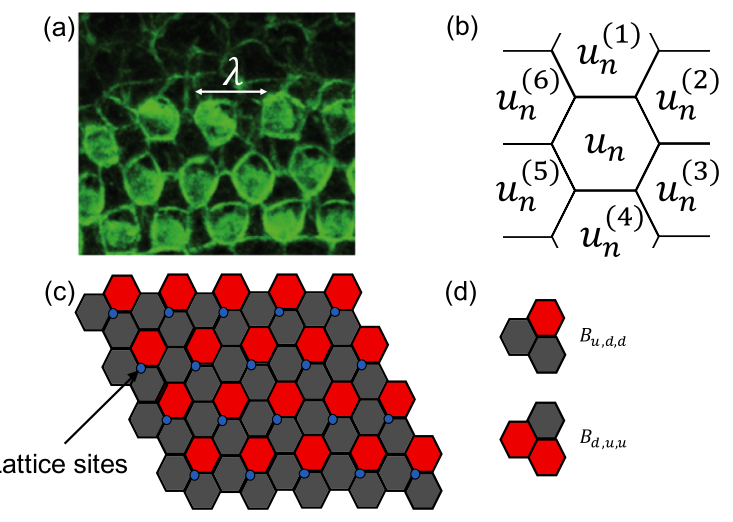

FIG. 1. Periodic fine-grained patterns. (a) Example from the auditory epithelium with a green fluorescent marker for actin adapted from Ref. [9], where $\lambda$ depicts the characteristic wavelength of the pattern. (b) Depiction of the notation used for Eq. (1). (c) Pattern in a hexagonal lattice, where at each lattice point (blue points) there is a basis structure with three cells, and (d) the possible basis structures for a periodic pattern.

where $x$ corresponds to the position in the line of cells and $\Delta x$ is the distance between the cells. A naïve stability analysis done on this continuum approximation, by linearizing Eq. (2) and using the test function $u(x, t)=u_{o}+\delta u(x, t)$, where $u_{o}=0, \delta u(x, t)=a_{k} e^{\alpha t+i k x}$ and $\Omega(x)=0$ would show that for lateral inhibition $(\epsilon<$ $0)$ the most unstable mode corresponds to $k=\infty$ with a growth rate $\alpha=\infty$ (Fig. 2). This is because neighboring cells alternate between positive and negative values and in the continuum approximation this corresponds to $k=\infty$.

In this work we determine the necessary conditions to obtain noisy anticorrelated patterns and periodic alternating patterns for $u_{n}$, given the signaling strength $(\epsilon)$ between cells and the profile of internal gene expression rate $\left(\Omega_{n}\right)$. We do this by making an analogy with optical phonons from solid-state physics [19], for which we need to represent the arrangement of cells in terms of a crystal. A crystal will be composed by lattice sites [blue points in Fig. 1(c)] and a

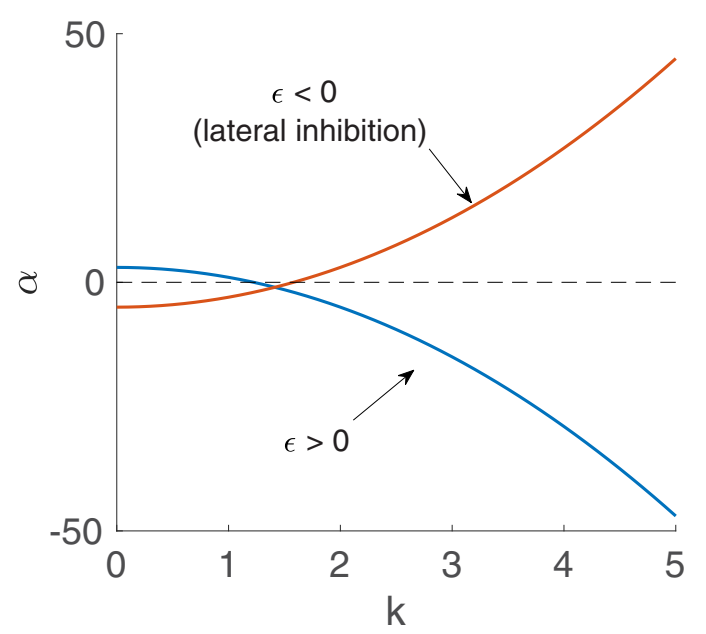

FIG. 2. Linear stability analysis of Eq. (2), where $k$ corresponds to the wave number and $\alpha$ is the mode growth rate.

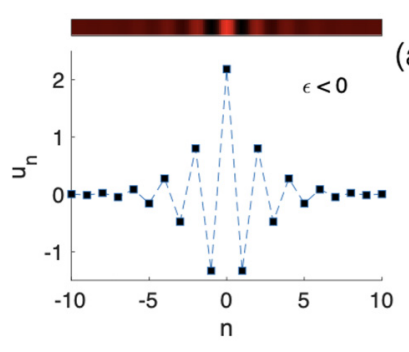

(a)
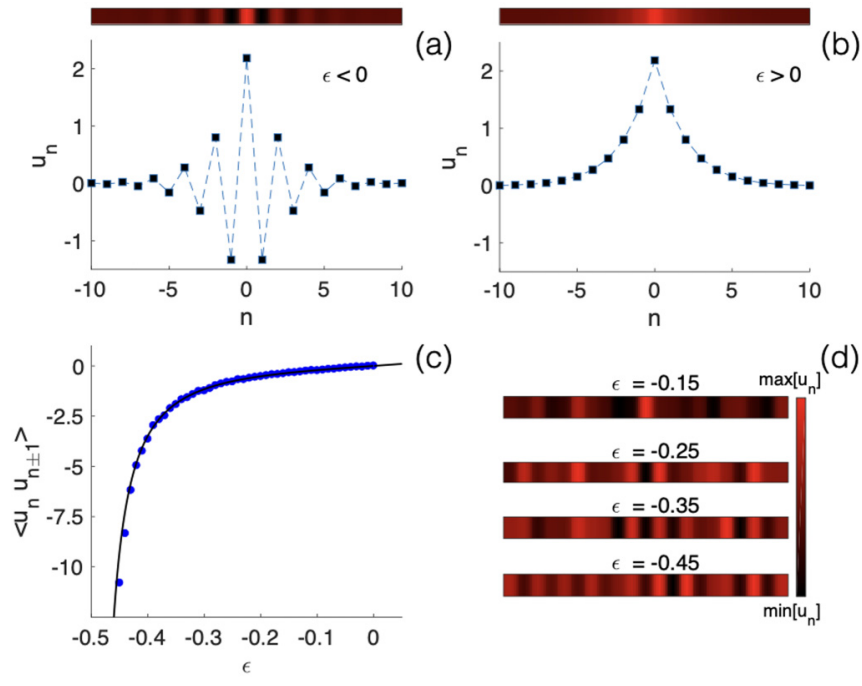

FIG. 3. Impulse response for a linear chain of cells. We set $\Omega_{n}=$ $\Omega_{o} \delta_{n 0}$, where (a) corresponds to $\epsilon<0$ and (b) to $\epsilon>0$ for a finite chain of 20 cells. Response to random input $\Omega_{n}$, where (c) shows the next-neighbor correlations $\left\langle u_{n} u_{n \pm 1}\right\rangle$ as a function of $\epsilon$, symbols correspond to numerical simulations, and solid curve was obtained analytically [Eq. (6)], and (d) examples of noisy anticorrelated patterns as a function of $\epsilon$.

structure called the basis of the crystal [Fig. 1(d)]. The basis is the repeating unit that is placed at each lattice site [see Fig. 1(c)]. The case of a one-dimensional chain of cells is explicitly solved, and these results helps us to analyze the case of cells placed in a hexagonal lattice. For the linear chain of cells the basis is composed by two cells and the redefined lattice sites correspond also to a linear chain. For cells placed in a hexagonal lattice, the basis is composed of three cells [see Fig. 1(d)] and the redefined lattice sites is also a hexagonal lattice. In cells arranged in a hexagonal lattice we obtain (1) a periodic pattern filled with the basis structure denoted $B_{u, d, d}$ [Fig. 1(d)], (2) a pattern filled with the basis $B_{d, u, u}$ [Fig. 1(d)], or (3) a state where both $B_{u, d, d}$ and $B_{d, u, u}$ coexist. Finally, we observed that if the system is driven from a periodic pattern filled with one basis to a pattern filled with the other basis, hysteresis is observed. This provides new predictions for experiments.

\section{LINEAR REPONSE OF A CHAIN OF CELLS}

We examine a one-dimensional chain of cells signaling each other following Eq. (1). For this example, we define a cell in the middle of the chain with index $n=0$. Consider the linearized version of Eq. (1) around $u_{n}=0$, which is given by

$$
\frac{d u_{n}}{d t} \approx-u_{n}+\epsilon\left[u_{n-1}+u_{n+1}\right]+\Omega_{n} .
$$

As a first step to characterize its dynamics we study the linear response function of the chain to the static input $\Omega_{n}=\Omega_{o} \delta_{n 0}$, where $\delta_{n n^{\prime}}$ corresponds to the Kronecker $\delta$. The linear response of an infinite chain is given by the following 
expression:

$$
u_{n}=\frac{\Omega_{o}}{\sqrt{1-4 \epsilon^{2}}}\left[\frac{1-\sqrt{1-4 \epsilon^{2}}}{2 \epsilon}\right]^{|n|}
$$

(see Appendix A for details). Note in Eq. (4) that there is a term proportional to $\epsilon^{-|n|}$; therefore, if $\epsilon<0$, then the values of $u_{n}$ alternate between positive and negative values [Fig. 3(a), lateral inhibition], while for $\epsilon>0$ the values of $u_{n}$ decay smoothly [Fig. 3(b), lateral induction].

We next calculate the correlations in a chain of $u_{n}$ now considering a stochastic input $\Omega_{n}$ characterized by

$$
\left\langle\Omega_{n} \Omega_{n^{\prime}}\right\rangle=\sigma_{\Omega}^{2} \delta_{n n^{\prime}} .
$$

The linear response function is used to calculate the correlation between nearest neighbors (see Appendix B), which is given by

$$
\left\langle u_{n} u_{n \pm 1}\right\rangle \approx \frac{2 \sigma_{\Omega}^{2}}{\epsilon}\left(1-\frac{1}{\sqrt{1-4 \epsilon^{2}}}\right)^{2}
$$

The correlations are zero for $\epsilon=0$ and they diverge to $-\infty$ for $\epsilon=-1 / 2$; this is the same value found in Collier et al. [13] by linear stability analysis. The divergence in the correlation coefficient is characteristic of a critical point [20], while its negative sign denotes that the neighbors are anticorrelated. At this critical point $\left(\epsilon=\epsilon_{c}\right)$ the uniform state $u_{n}=0$ becomes unstable. There is good agreement between numerical and the analytical approximation given by Eq. (6) [see Fig. 3(c)]. Examples of anticorrelated noisy patterns in Fig. 3(d) show that as $\epsilon$ decreases, the probability of having alternating neighbors increases.

\section{ANALOGY TO OPTICAL MODES}

The results shown in Figs. 3(a) and 3(b) are reminiscent of the optical and acoustic modes of phonons from solid-state physics [19]. Phonons are vibrations in a periodic crystal. The crystal is composed by a set of lattice sites, where at each site there is a molecule with several atoms called the basis (also termed as the crystal unit cell). If the basis is diatomic, then two vibration modes are allowed. In the optical mode the position of atoms alternate from positive to negative values between neighbors, and in the acoustic mode this alternation does not happen [19]. The linear response of $u_{n}$ for $\epsilon<0$ [Fig. 3(a)] is analogous to the optical mode and for $\epsilon>0$ to the acoustic mode [Fig. 3(b)].

This analogy to the optical modes is useful to analyze the subcritical state $\left(\epsilon<\epsilon_{c}\right)$. We start by setting a uniform $\Omega_{n}=$ $\Omega_{o}$ in Eq. (1). We redefine the chain of $u_{n}$ into $s$ lattice sites and at each site there is a basis with two components $v_{s}$ and $w_{s}$ [Fig. 4(a)]. The components $v_{s}$ corresponds to $u_{n}$ with $n=0$ and even values of $n$, and the remainder corresponds to $w_{s}$. In this notation, Eq. (1) for the one-dimensional chain becomes

$$
\begin{aligned}
& \frac{d v_{s}}{d t}=-v_{s}-\gamma v_{s}^{3}+\epsilon\left[w_{s-1}+w_{s}\right]+\Omega_{o}, \\
& \frac{d w_{s}}{d t}=-w_{s}-\gamma w_{s}^{3}+\epsilon\left[v_{s}+v_{s+1}\right]+\Omega_{o} .
\end{aligned}
$$

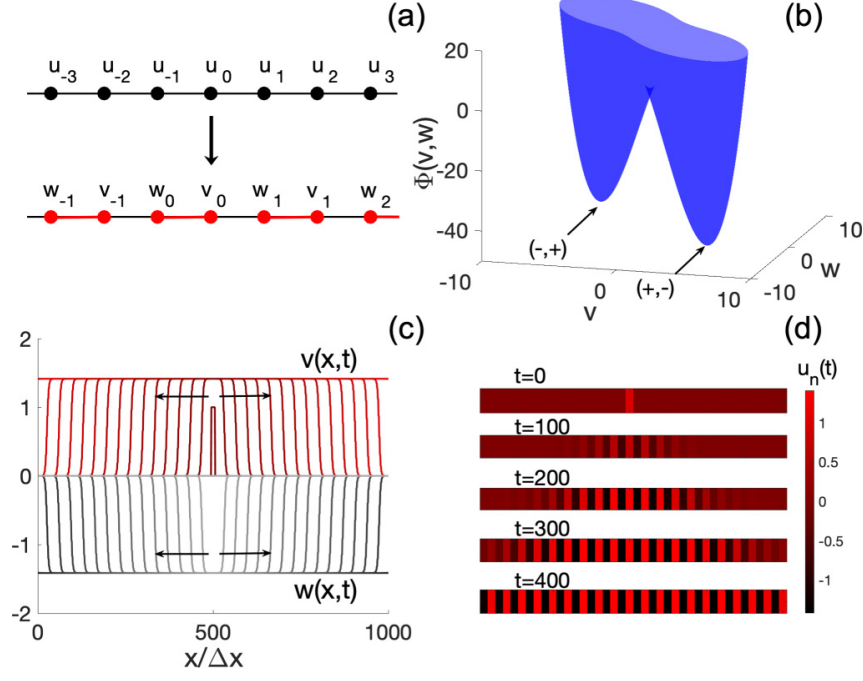

FIG. 4. Potential well for a linear chain of cells. (a) Redefinition of the crystal basis from a single-component basis $u$ to a twocomponent basis $u, v$ (diatomic). (b) An example of a potential $\Phi(v, w)$ with $\epsilon<1 / 2$. The potential has minima at $(+,-)$ (positive $v_{s}$ and negative $w_{s}$ ) and $(-,+)$ (negative $v_{s}$ and positive $w_{s}$ ). (c) Time evolution of the continuum approximation of $v(x, t)$ and $w(x, t)$ defined by Eqs. (7) and (8) and (d) time evolution of the chain of $u_{n}$ given by Eq. (1).

We rewrite Eqs. (7) and (8) in the continuum approximation as

$$
\begin{aligned}
& \frac{\partial v}{\partial t}=2 \epsilon w-v-\gamma v^{3}+\epsilon \Delta x^{2} \frac{\partial^{2} w}{\partial x^{2}}+\Omega_{o}, \\
& \frac{\partial w}{\partial t}=2 \epsilon v-w-\gamma w^{3}+\epsilon \Delta x^{2} \frac{\partial^{2} v}{\partial x^{2}}+\Omega_{o},
\end{aligned}
$$

where $x$ corresponds to the position in the line tissue and $\Delta x$ is the distance between the lattice points. Assuming a uniform stationary state for both $v_{s}$ and $w_{s}$, we then define a potential

$$
\Phi(v, w)=-\Omega_{o}(v+w)+\frac{v^{2}+w^{2}}{2}-2 \epsilon v w+\gamma \frac{v^{4}+w^{4}}{4}
$$

for $\epsilon<\epsilon_{c}$ this is a double-well potential [Fig. 4(b)]. One minimum of this potential we denote as the state $(+,-)$ where $v_{s}=c_{o}$ and $w_{s}=-c_{o}$. The other minimum we denote as $(-,+)$, where $v_{s}=-c_{o}$ and $w_{s}=c_{o}$. From this potential we expect that the steady states of Eq. (1) consists of anticorrelated neighbors.

To test this prediction we first simulated the continuum approximation given by Eqs. (9) and (10) with periodic boundary conditions and $\Omega(x)=0$. We have set the initial conditions to $v(x, 0)=\theta\left(x-x_{1}\right)-\theta\left(x-x_{2}\right)$ and $w(x, 0)=0$, where $\theta(x)$ corresponds to the Heaviside function [Fig. 4(c)]. Note that $v(x, 0)$ evolves into two fronts going to the sides, the fronts finally settle to the value $v(x, t)=c_{o}$, while $w(x, 0)$ evolves also into two fronts that are anticorrelated to those of $v(x, t)$ until they settle to the value $w(x, t)=-c_{o}$. This confirms the steady states shown in the potential of Fig. 4(b). Finally, we simulate Eq. (1) for a linear chain of 20 cells with periodic boundary conditions, setting the initial condition 
(a)

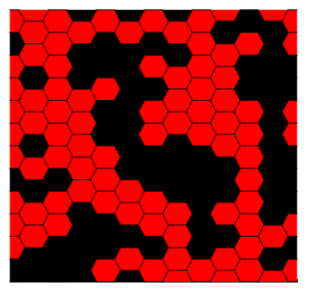

(b)

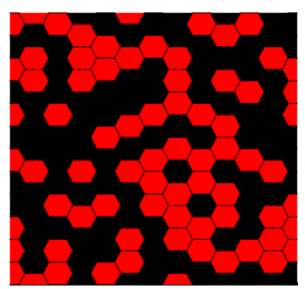

(c)

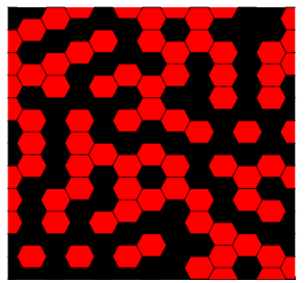

(d)

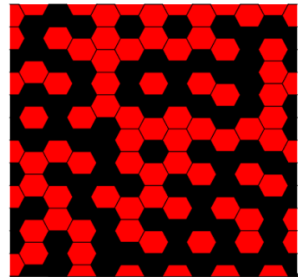

FIG. 5. Increase in cell anticorrelation with increasing coupling strength. Stochastic patterns given by $f_{n}=2 \theta\left(u_{n}\right)-1$ generated with noisy input $\Omega_{n}$, where black corresponds to $f_{n}=-1$ and red to $f_{n}=1$. (a) $\epsilon=0$, (b) $\epsilon=-0.1$, (c) $\epsilon=-0.2$, and (d) $\epsilon=-0.3333$.

to $u_{n}(t)=\delta_{n, 0}$. In the same way as in the continuum approximation, two fronts going to the sides develop. The cells settle to an alternating pattern where cells with label $v_{s}=c_{o}$ and cells with label $w_{s}=-c_{o}$. These fronts are reminiscent of waves observed before in the Collier model [21].

As mentioned in the Introduction, naive linear stability analysis of Eq. (2) shows that the most unstable mode is $k=\infty$. By redefining the cell arrangement in terms of lattice sites and basis $v$ and $w$ we determine that the most unstable mode is actually $k=0$ (the $\Gamma$ point in optical phonons). From this analysis the basis $v$ and $w$ is bistable due to coupling

by lateral inhibition. In the literature there are two types of lateral inhibition models, one in which the system becomes unstable due to cell-to-cell coupling, as shown here and in Refs. [11,13,14], and another where single cells are intrinsically bistable [22]. The calculations for bistable systems are straightforward and the minima of the potential are also anti-correlated (see Appendix C).

\section{CELLS IN AN HEXAGONAL LATTICE}

Typical fine-grained pattern formation occurs in epithelial tissues, single 2D layers of cells. As a good approximation of these tissues, we analyze the case of cells placed in a hexagonal lattice. Using the observations of a linear chain of cells we analyze the response in $u_{n}$ to a random input $\Omega_{n}$ in epithelial tissues. Figure 5 shows the projection $f_{n}=$ $2 \theta\left(u_{n}\right)-1$ for different steady states as a function of $\epsilon$. As in the example of the previous section, we see that the likelihood of being surrounded by anticorrelated neighbors increases as the value of $\epsilon$ decreases.

We proceed by analyzing the subcritical regime of Eq. (1) by dividing $u_{n}$ into different components as in the previous example. Again we consider a spatially uniform $\Omega_{n}=\Omega_{o}$. Defining a basis with two variables as in the linear chain, and rewriting Eq. (1), we notice that the interactions are asymmetric. We redefine a basis in terms of three components $v, w$, and $z$ [Fig. 6(a)]. This basis is embedded in another hexagonal lattice. In terms of lattice sites $\left\{s, s^{\prime}\right\}$ Eq. (1) becomes

$$
\begin{aligned}
\frac{d v_{s, s^{\prime}}}{d t}= & -v_{s, s^{\prime}}-\gamma v_{s, s^{\prime}}^{3}+\epsilon\left[w_{s-1, s^{\prime}}+w_{s, s^{\prime}}+w_{s, s^{\prime}+1}\right. \\
& \left.+z_{s-1, s^{\prime}-1}+z_{s-1, s^{\prime}}+z_{s, s^{\prime}}\right]+\Omega_{o},
\end{aligned}
$$

(a)
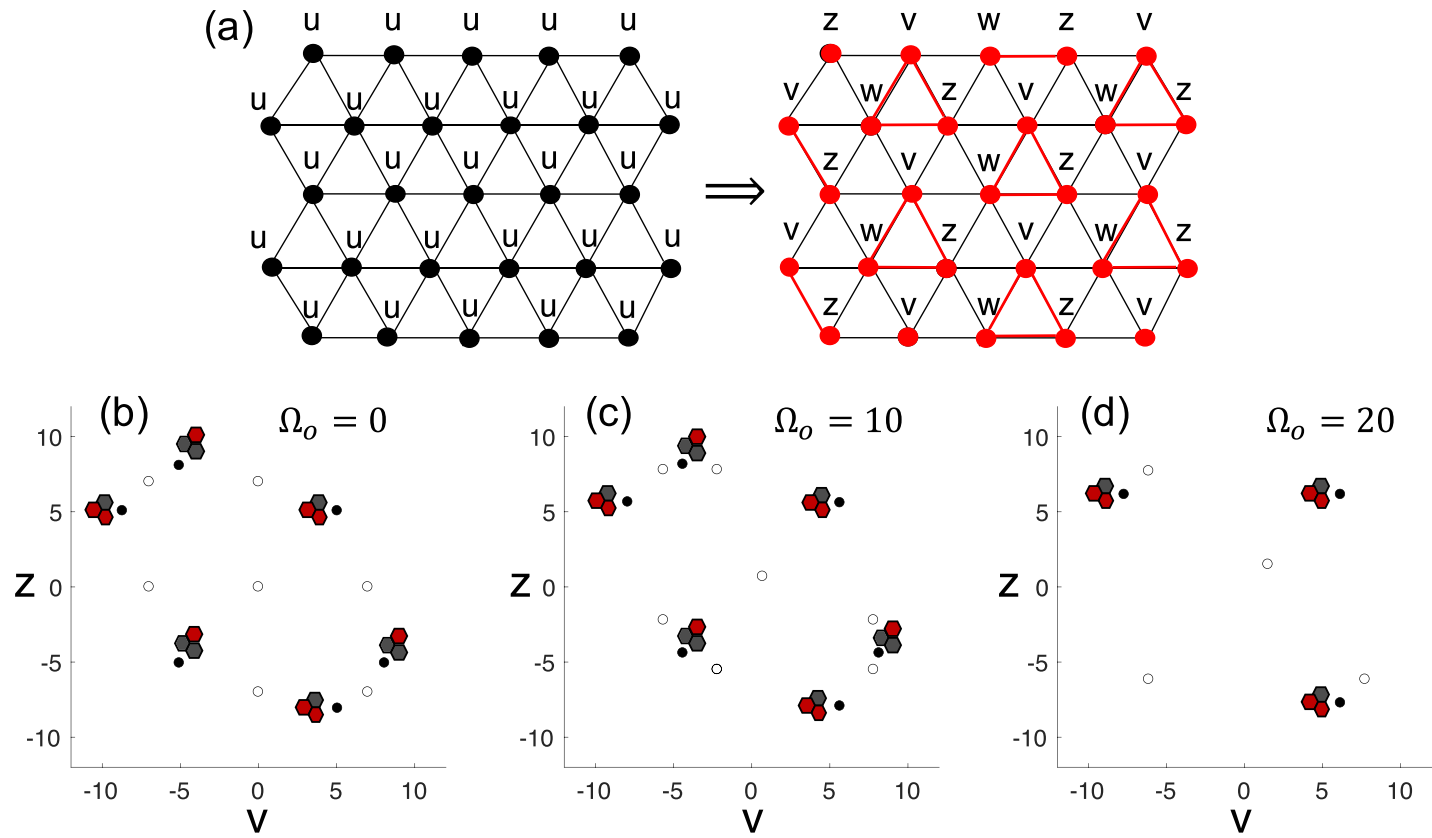

FIG. 6. Redefining the basis and metastable and stable points of $\Phi(v, w, z)$. (a) Redefinition of the crystal basis from a single-component (u) to a three-component basis $(v, w, z)$ in hexagonal lattices. [(b) and (c)] Stable states (black symbols) and metastable states (white symbols) of the potential $\Phi(v, w, z)$ given by Eq. (18) projected onto the $v-z$ axis for the parameters $\gamma=0.1$ and $\epsilon=-2$. 


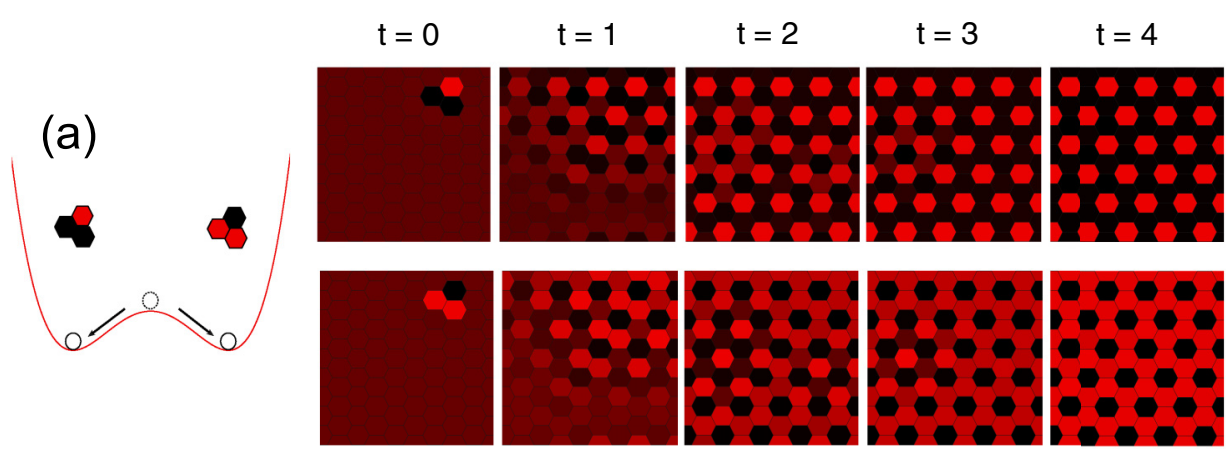

(b)

(c)
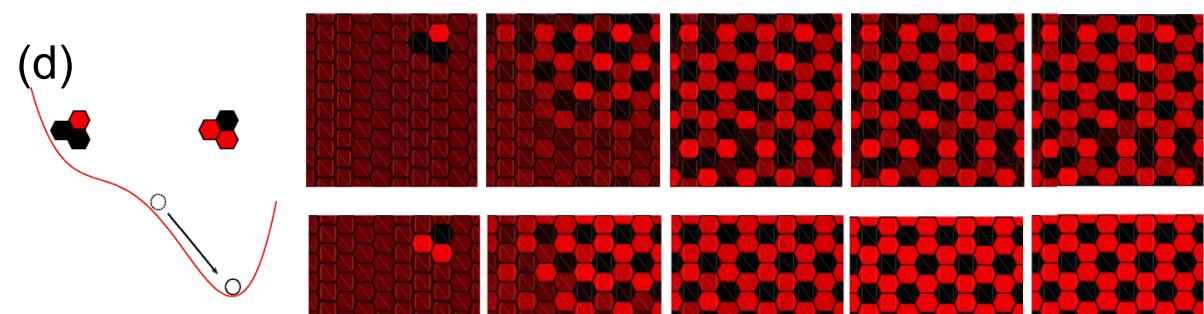

(e)
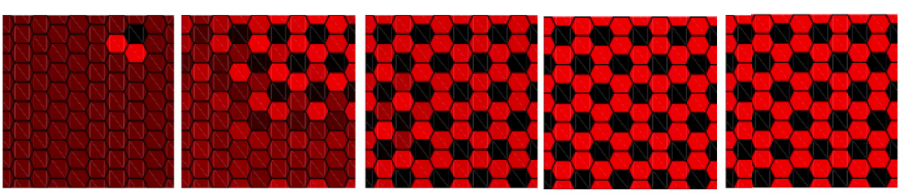

(f)
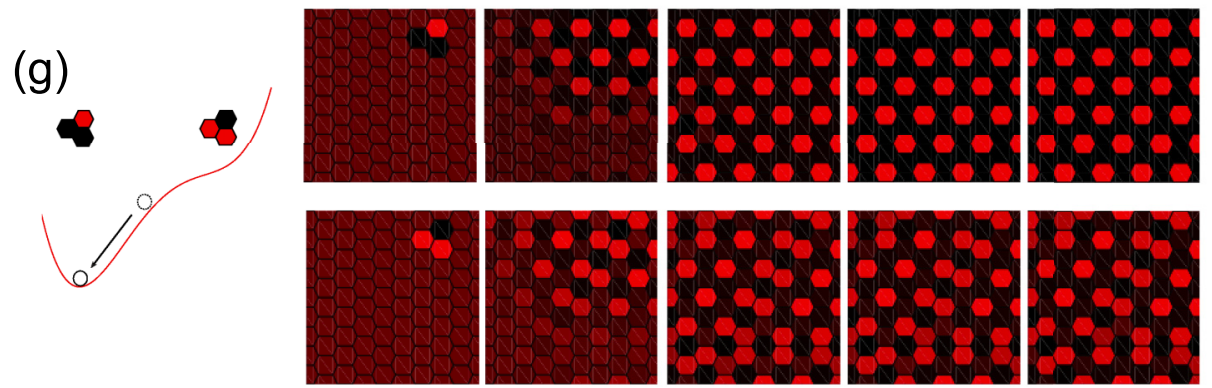

(h)

FIG. 7. Conceptual double-well potential and corresponding pattern selection. (a) Double-well potential at $\Omega_{o}=0$, where the minima represents the stability of the basis $B_{u, d, d}$ and $B_{d, u, u}$, and [(b) and (c)] simulations initiated with uniform initial conditions $u_{n, n^{\prime}}=0$ except for three cells set to (b) $B_{u, d, d}$ and (c) $B_{d, u, u}$. In this regime the final pattern supports a periodic repetition of either basis. For $\Omega_{o}>\Omega_{c}$, (d) the single-well potential shows the basis $B_{d, u, u}$ is stable. Simulations initiated with (e) $B_{u, d, d}$ and (f) $B_{d, u, u}$, show that the final pattern supports only the basis $B_{d, u, u}$. For $\Omega_{o}<\Omega_{c}$, (g) the single-well potential shows the basis $B_{u, d, d}$ is stable. Simulations initiated with (h) $B_{u, d, d}$ and (i) $B_{d, u, u}$ show that the final pattern supports only the basis $B_{u, d, d}$.

$$
\begin{gathered}
\frac{d w_{s, s^{\prime}}}{d t}=-w_{s, s^{\prime}}-\gamma w_{s, s^{\prime}}^{3}+\epsilon\left[v_{s, s^{\prime}-1}+v_{s, s^{\prime}}+v_{s+1, s^{\prime}}\right. \\
\left.+z_{s-1, s^{\prime}-1}+z_{s, s^{\prime}-1}+z_{s, s^{\prime}}\right]+\Omega_{o} \\
\frac{d z_{s, s^{\prime}}}{d t}=-z_{s, s^{\prime}}-\gamma z_{s, s^{\prime}}^{3}+\epsilon\left[v_{s, s^{\prime}}+v_{s+1, s^{\prime}}+v_{s+1, s^{\prime}+1}+w_{s, s^{\prime}}\right. \\
\left.+w_{s, s^{\prime}+1}+w_{s+1, s^{\prime}+1}\right]+\Omega_{o} .
\end{gathered}
$$

If we perform a Taylor expansion to rewrite Eqs. (12)-(14) as a spatial temporal system we find

$$
\begin{aligned}
& \frac{\partial v}{\partial t}=3 \epsilon[w+z]-v-\gamma v^{3}+\Omega_{o}+\epsilon S(w, z), \\
& \frac{\partial w}{\partial t}=3 \epsilon[v+z]-w-\gamma w^{3}+\Omega_{o}+\epsilon S(v, z), \\
& \frac{\partial z}{\partial t}=3 \epsilon[v+w]-z-\gamma z^{3}+\Omega_{o}+\epsilon S(v, w),
\end{aligned}
$$

where $S$ corresponds to the terms involving spatial derivatives. Assuming that at steady state this continuum system settles to $k=0$, then also $S=0$. Then we can define the following potential:

$$
\begin{aligned}
\Phi(v, w, z)= & -\Omega_{o}(v+w+z)+\frac{v^{2}+w^{2}+z^{2}}{2} \\
& -3 \epsilon[v w+v z+w z]+\gamma \frac{v^{4}+w^{4}+z^{4}}{4} .
\end{aligned}
$$

The state of the tissue becomes unstable at $\epsilon_{c}=-1 / 3$ [13], and in this case we find the minima of this potential and their stability numerically. Figures 6(b)-6(d) show the position of the stable (black points) and the metastable states (white points) projected onto the $v-z$ axis from the potential given by Eq. (18) as a function of $\Omega_{o}$ for $\epsilon<\epsilon_{c}$. When $\Omega_{o}=0$ there are six stable states; three correspond to the basis $B_{u, d, d}$ [Fig. 6(b)] mentioned in the Introduction [see Fig. 1(d)]. The 

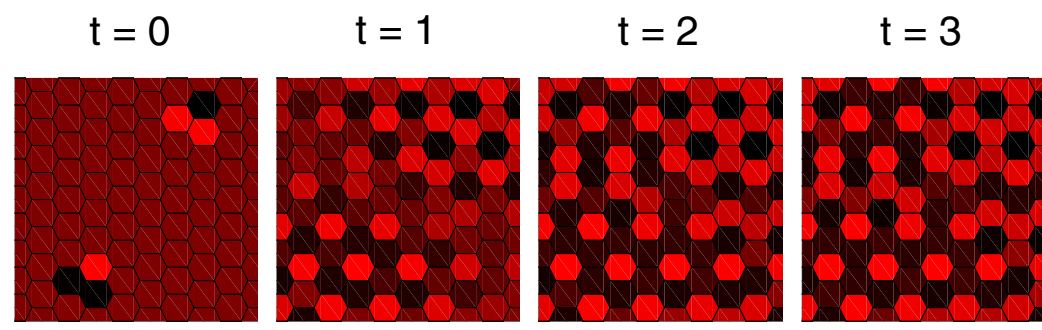

$$
t=4
$$

FIG. 8. Coexistence of both base at $\Omega_{o}=0$. A simulation is initiated with uniform initial conditions and three cells forming the basis $B_{u, d, d}$ and three cells forming $B_{d, u, u}$. At the end $(t=4)$ the stable pattern contains clusters of both base which meet at interfaces.

other stable states correspond to the basis $B_{d, u, u}$. A simplified picture is shown in Fig. 7(a) as a double-well potential where each minima corresponds to a basis. In Fig. 7(b) we show a numerical simulation of Eq. (1) for periodic boundary conditions. A $t=0$ the initial conditions are uniform with $u_{n}=0$ except for three cells that are set with the basis $B_{u, d, d}$. We see that as time passes the tissue is filled with the basis $B_{u, d, d}$ until it makes a periodic pattern. The pattern is characterized with cells having $u_{s, s^{\prime}}>0$ being surrounded with six cells with $u_{s, s^{\prime}}<0$. We repeated the simulation, but now the initial three cells are set with the basis $B_{d, u, u}$ [Fig. 7(c)]. Again, the pattern becomes periodic but cells with $u_{s, s^{\prime}}<0$ are surrounded with six cells with $u_{s, s^{\prime}}>0$.

If we increase further the value of $\Omega_{o}>0$ [Fig. 6(c)], the points indicating the metastable states move closer to the points indicating the basis $B_{u, d, d}$. After crossing a critical value for $\Omega_{c}$, the stable points corresponding to $B_{u, d, d}$ disappear by colliding with a metastable point [Fig. 6(d)]. By increasing the parameter $\Omega_{o}$ the double-well potential [Fig. 7(a)] becomes a single-well potential [Fig. 7(d)], corresponding to the basis $B_{d, u, u}$. We simulate Eq. (1) in this regime and with the same initial conditions as in Figs. 7(b) and 7(c); if the simulation is initiated with the basis $B_{u, d, d}$ [Fig. 7(e)], then we see that the final pattern is filled with $B_{d, u, u}$. The pattern is not periodic due to a defect that arises from the initial

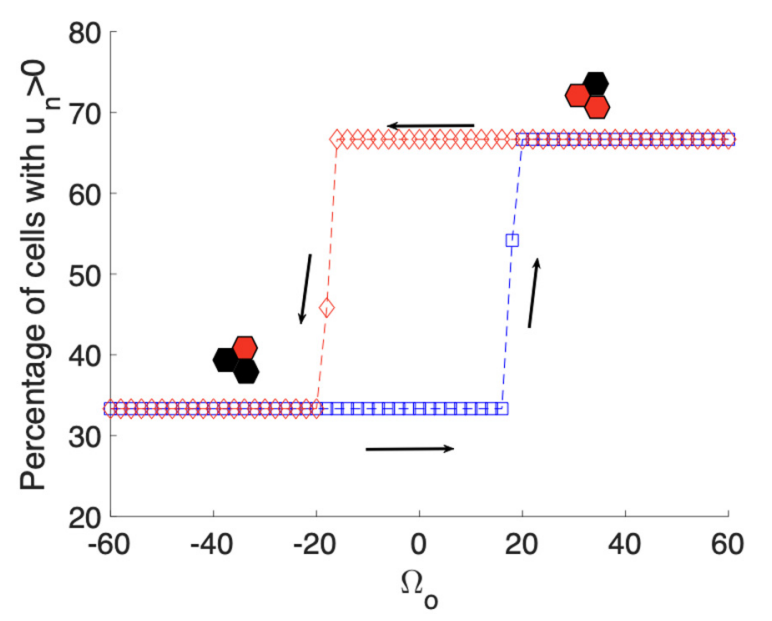

FIG. 9. Hysteresis curve between patterns. The system is initiated with a pattern filled with the basis $B_{u, d, d}$ and the parameter $\Omega_{o}$ is swept back and forth between -60 and 60 . The lower branch corresponds to a pattern filled with the basis $B_{u, d, d}$ and the upper branch to $B_{d, u, u}$. conditions. On the other hand, if the simulation is initiated with the basis $B_{u, d, d}$ Fig. 7(f)], then the final pattern is periodic and filled with the basis $B_{u, d, d}$. With these initial conditions we get the same pattern as in Fig. 7(c). For the opposite case where $\Omega_{0}<-\Omega_{c}$ [Fig. $7(\mathrm{~g})$ ], we observe a periodic pattern if initiated with the basis $B_{u, d, d}$ [Fig. 7(h)] and defects if initiated with $B_{d, u, u}$ [Fig. 7(i)]. From these results we see that by redefining the basis in hexagonal lattices in terms of $v, w$, and $z$, the most unstable mode is $k=0$.

\section{EXPERIMENTAL PREDICTIONS}

Finally, we discuss two experimental predictions. First, we notice that at $\Omega_{o}=0$ both bases can coexist. We simulated this regime by setting the initial conditions with three cells with the basis $B_{u, d, d}$ and another three cells with the basis $B_{d, u, u}$ (see Fig. 8 at $t=0$ ) while all others are set to $u_{s, s^{\prime}}=0$. As time evolves we see that the final pattern is filled with both bases that meet at different interfaces. As mentioned in the Introduction, the parameter $\Omega_{o}$ is equivalent to the gene expression rate of active Delta. It is possible that this state with two coexisting bases can be reached in experiment by modulating the amount of active Delta in cells.

Second, due to the multistability of the potential of $\Phi(v, w, z)$ [Eq. (18)], it might be possible to observe hysteresis in the system. In Fig. 9 we show hysteresis curve, where we quantified the percentage of cells that showed $u_{s, s^{\prime}}>0$ given the value of $\Omega_{o}$. Starting with a pattern filled with $B_{u, d, d}$, noting as $\Omega_{o}$ changes there is a wide range of values where $B_{u, d, d}$ is stable, until it reaches a critical point where it becomes unstable and the transition $B_{u, d, d} \rightarrow B_{d, u, u}$ is observed. A further increase of this parameter changes nothing. After that, if we decrease $\Omega_{o}$ at some critical point we observe the transition $B_{d, u, u} \rightarrow B_{u, d, d}$. The critical points for the transition $B_{u, d, d} \rightarrow B_{d, u, u}$ and $B_{d, u, u} \rightarrow B_{u, d, d}$ are not at the same position, meaning that the tissue shows memory. Again this might be observed in experiment by changing the amount of active Delta.

\section{CONCLUSIONS}

In this work we have proposed an analytically tractable model for lateral inhibition. We have shown how fine-grained checkerboard patterns depend on the model parameters. By making an analogy with optical modes from lattice phonons we are able to determine how fine-grained patterns are selected. Thus our model is a generic description of lateral inhi- 
bition that explains the transitions observed in more detailed models [13-18].

We note that our model is a completely different mechanism from that which produces hexagonal patterns in reactiondiffusion systems. A canonical model that creates such patterns is the Swift-Hohenberg equation with non-Boussinesq conditions [23]. A reaction-diffusion with two components can approximate the Swift-Hohenberg equation [24], in contrast to our system, which is a single-component model. The Swift-Hohenberg equation is characterized by an instability around a central mode $k=k_{c}$. Here we have shown that the most unstable mode in lateral inhibition is $k=\infty$ (Fig. 2). By redefining the lattice structure and the basis of the cells, the system behaves effectively as a multicomponent system where the most unstable mode is $k=0$. In terms of lattice vibrations this corresponds to an optical phonon at the $\Gamma$ point.

Our model brings a new framework to studying and understanding pattern selection in both natural and synthetic biological fine-grained systems. Particularly attractive experimental scenarios for testing these ideas are in vitro cellular systems with synthetic intercellular signaling pathways such as recently reported by Matsuda et al. [25]. Disrupting DeltaNotch communication between cells in this system using a chemical inhibitor yielded a uniform expression of Delta across the tissue. Removal of the inhibitor allowed DeltaNotch signaling to initiate and caused the evolution of a finegrained pattern of Delta expression. This mimics our model's behavior when the coupling strength is modulated from $\epsilon=0$ to $\epsilon<\epsilon_{c}$. This and similar experimental systems [26,27] also raise the possibility of controlling the initial expression levels of Delta, or an equivalent signal, which would be analogous to modulating $\Omega_{o}$ in the model. Our analysis makes the experimental prediction that by modulating the expression levels a system with lateral inhibition can be driven to a regime where the two basis coexist or show hysteresis.

Finally, our analysis suggests that embryonic pattern formation by local cell signaling has two regimes. A regime where the steady state is composed by acoustic modes, this is the reaction-diffusion scenario proposed by Turing and a regime composed by optical modes that corresponds to the fine-grained patterns of lateral inhibition.

\section{ACKNOWLEDGMENTS}

We thank Marta Ibañes and José M. Sancho for sharing their unpublished manuscript featuring a similar model. Their analysis and observations are complementary to our work. This work was funded through institutional support from EPFL and a SNF project Grant No. 31003A_176037.

\section{APPENDIX A: RESPONSE FOR A LINEAR CHAIN OF CELLS}

The first step to calculate the steady state for a linear chain of cells is finding the response for $u_{0}$, which corresponds to the cell at the center of the chain. We do this recursively by solving explicitly the case of finite chains. The steady state of the chain is given by

$$
-u_{n}+\epsilon\left[u_{n-1}+u_{n+1}\right]+\Omega_{o} \delta_{n, 0}=0,
$$

and we impose a condition for symmetry around $u_{0}$ such that we can rewrite Eq. (A1) for $n=0$ as

$$
-u_{0}+2 \epsilon u_{ \pm 1}+\Omega_{o}=0
$$

and for the rest of the chain

$$
-u_{n}+\epsilon\left[u_{n-1}+u_{n+1}\right]=0 .
$$

The solutions for chains with five, seven, and nine cells are given, respectively, by

$$
\begin{gathered}
\frac{\Omega_{o}}{u_{0}}=1-\frac{2 \epsilon^{2}}{1-\epsilon^{2}}, \\
\frac{\Omega_{o}}{u_{0}}=1-\frac{2 \epsilon^{2}}{1-\frac{\epsilon^{2}}{1-\epsilon^{2}}}, \\
\frac{\Omega_{o}}{u_{0}}=1-\frac{2 \epsilon^{2}}{1-\frac{\epsilon^{2}}{1-\frac{\epsilon^{2}}{1-\epsilon^{2}}}},
\end{gathered}
$$

and therefore we can infer for an infinite chain that the response is given by

$$
\frac{\Omega_{o}}{u_{0}}=1-\frac{2 \epsilon^{2}}{1-\frac{\epsilon^{2}}{1-\frac{\epsilon^{2}}{1-\ldots}}} .
$$

Due to the periodicity of the fraction we can rewrite it as

$$
\frac{\Omega_{o}}{u_{0}}=1-\frac{2 \epsilon^{2}}{1-\frac{1-\Omega_{o} / u_{0}}{2}},
$$

and then the response of the cell in the middle is given by

$$
u_{0}=\frac{\Omega_{o}}{\sqrt{1-4 \epsilon^{2}}} .
$$

Inserting the solution given by Eq. (A9) into Eqs. (A1) and (A2) we find

$$
u_{n}=\frac{\Omega_{o}}{\sqrt{1-4 \epsilon^{2}}}\left[\frac{1-\sqrt{1-4 \epsilon^{2}}}{2 \epsilon}\right]^{|n|} .
$$

\section{APPENDIX B: NEXT-NEIGHBOR CORRELATIONS FOR A LINEAR CHAIN OF CELLS}

We rewrite the responses for $\Omega_{n}=\Omega_{o} \delta_{n, 0}$ as $u_{n}=\Omega_{0} G_{n}$, where $G_{n}$ are analogous to Green functions. Suppose that now the chain is forced by an input $\Omega_{n}$ with the following spatial correlation:

$$
\left\langle\Omega_{n} \Omega_{n^{\prime}}\right\rangle=\sigma_{\Omega}^{2} \delta_{n, n^{\prime}},
$$

then the response of the chain is given by the convolution $u_{n}=$ $G_{n} * \Omega_{n}$. The correlations between two cells in the chain is given by

$$
\left\langle u_{n} u_{n^{\prime}}\right\rangle=2 \sigma_{\Omega}^{2} \sum_{m=0}^{\infty} G_{n+m} G_{n^{\prime}+m},
$$

this expression is easily simplified by rewriting Eq. (A1) in terms of $G_{n}$; by multiplying it by $G_{n}$ we find

$$
\frac{G_{n}^{2}-\delta_{n, 0} G_{n}}{\epsilon}=G_{n-1} G_{n}+G_{n+1} G_{n},
$$


and if we set $n=0$ and $n^{\prime}=1$, then their correlations can be written as

$$
\left\langle u_{0} u_{1}\right\rangle=2 \sigma_{\Omega}^{2} \sum_{m=0}^{\infty} G_{2 m+1}^{2} .
$$

Using only the first term in the sum we get the approximation

$$
\left\langle u_{0} u_{1}\right\rangle \approx \frac{2 \sigma_{\Omega}^{2}}{\epsilon}\left(1-\frac{1}{\sqrt{1-4 \epsilon^{2}}}\right)^{2} .
$$

\section{APPENDIX C: EXTENSION TO BISTABLE SYSTEMS}

The extension of Eq. (1) to the bistable case is given by

$$
\frac{d u_{n}}{d t}=\alpha u_{n}-\gamma u_{n}^{3}+\Omega_{n}+\epsilon \sum_{i=1}^{N} u_{n}^{(i)}
$$

where $\alpha>0$ makes the units bistable. For the linear lattice the equivalent to Eqs. (7) and (8) are

$$
\begin{aligned}
& \frac{\partial v}{\partial t}=2 \epsilon w+\alpha v-\gamma v^{3}+\epsilon \Delta x^{2} \frac{\partial^{2} w}{\partial x^{2}}+\Omega_{o}, \\
& \frac{\partial w}{\partial t}=2 \epsilon v+\alpha w-\gamma w^{3}+\epsilon \Delta x^{2} \frac{\partial^{2} v}{\partial x^{2}}+\Omega_{o},
\end{aligned}
$$

and the potential for this case corresponds to

$$
\Phi(v, w)=-\Omega_{o}(v+w)-\alpha \frac{v^{2}+w^{2}}{2}-2 \epsilon v w+\gamma \frac{v^{4}+w^{4}}{4} .
$$

A quick parameter sweep shows that there is also a doublewell potential where $v$ and $w$ are also anticorrelated.
[1] A. M. Turing, Philos. Trans. R. Soc. B 237, 37 (1952).

[2] V. Castets, E. Dulos, J. Boissonade, and P. De Kepper, Phys. Rev. Lett. 64, 2953 (1990).

[3] Q. Ouyang and H. Swinney, Nature 352, 610 (1991).

[4] M. Cross and H. Greenside, Pattern Formation and Dynamics in Nonequilibrium Systems (Cambridge University Press, Cambridge, 2009).

[5] S. Kondo and R. Asai, Nature 376, 765 (1995).

[6] M. Yamaguchi, E. Yoshimoto, and S. Kondo, Proc. Natl. Acad. Sci. USA 104, 4790 (2007).

[7] J. Raspopovic, L. Marcon, L. Russo, and J. Sharpe, Science 345, 566 (2014).

[8] A. Gavish, A. Shwartz, A. Weizman, E. Schejter, B-Z. Shilo, and N. Barkai, Nat. Commun. 7, 10461 (2015).

[9] H. Togashi, K. Kominami, M. Waseda, H. Komura, J. Miyoshi, M. Takeichi, and Y. Takai, Science 333, 1144 (2011).

[10] J. Adam, A. Myat, I. Le Roux, M. Eddison, D. Henrique, D. Ish-Horowicz, and J. Lewis, Development 125, 4645 (1998).

[11] F. Corson, L. Couturier, H. Rouault, K. Mazouani, and F. Schweisguth, Science 356, eaai7407 (2017).

[12] S. Artavanis-Tsakonas, K. Matsuno, and M. E. Fortini, Science 268, 225 (1995).

[13] J. R. Collier, N. A. Monk, P. K. Maini, and J. H. Lewis, J. Theor. Biol. 183, 429 (1996).
[14] D. Sprinzak, A. Lakhanpal, L. LeBon, J. Garcia-Ojalvo, and M. B. Elowitz, PLOS Comput. Biol. 7, e1002069 (2011).

[15] P. Formosa-Jordan and M. Ibañes, PLoS ONE 9, e95744 (2014).

[16] S. R. Veflingstad, E. Plahte, and N. A. Monk, Physica D 207, 254 (2005).

[17] H. Momiji and N. A. M. Monk, Phys. Rev. E 80, 021930 (2009).

[18] D. S. Glass, X. Jin, and I. H. Riedel-Kruse, Phys. Rev. Lett. 116, 128102 (2016).

[19] Charles Kittel, Introduction to Solid State Physics 8th ed. (John Wiley \& Sons, New York, 2004).

[20] M. Scheffer, J. Bascompte, W. A. Brock, V. Brovkin, S. R. Carpenter, V. Dakos, H. Held, E. H. van Nes, M. Rietkerk, and G. Sugihara, Nature 461, 53 (2009).

[21] E. Plahte and L. Øyehaug, Physica D 226, 117 (2006).

[22] D. K. Lubensky, M. W. Pennington, B. I. Shraiman, and N. E. Baker, Proc. Natl. Acad. Sci. USA 108, 11145 (2011).

[23] M. Bestehorn and H. Haken, Z. Phys. B 57, 329 (1984).

[24] M. C. Cross and P. C. Hohenberg, Rev. Mod. Phys. 65, 851 (1993).

[25] M. Matsuda, M. Koga, K. Woltjen, E. Nishida, and M. Ebisuya, Nat. Commun. 6, 6195 (2014).

[26] S. Toda, L. R. Blauch, S. K. Y. Tang, L. Morsut, and W. A. Lim, Science 361, 156 (2018).

[27] D. S. Glass and I. H. Riedel-Kruse, Cell 174, 649 (2018). 